\title{
Impact of Public Aggregate Wind Forecasts on Electricity Market Outcomes
}

\author{
Exizidis, Lazaros; Kazempour, Jalal; Pinson, Pierre; de Greve, Zacharie; Vallée, Francois
}

Published in:

IEEE Transactions on Sustainable Energy

Link to article, DOI:

10.1109/TSTE.2017.2682299

Publication date:

2017

Document Version

Peer reviewed version

Link back to DTU Orbit

Citation (APA):

Exizidis, L., Kazempour, J., Pinson, P., de Greve, Z., \& Vallée, F. (2017). Impact of Public Aggregate Wind Forecasts on Electricity Market Outcomes. IEEE Transactions on Sustainable Energy, 8(4), [7879306]. https://doi.org/10.1109/TSTE.2017.2682299

\section{General rights}

Copyright and moral rights for the publications made accessible in the public portal are retained by the authors and/or other copyright owners and it is a condition of accessing publications that users recognise and abide by the legal requirements associated with these rights.

- Users may download and print one copy of any publication from the public portal for the purpose of private study or research.

- You may not further distribute the material or use it for any profit-making activity or commercial gain

- You may freely distribute the URL identifying the publication in the public portal 


\title{
Impact of Public Aggregate Wind Forecasts on Electricity Market Outcomes
}

\author{
Lazaros Exizidis, Student Member, IEEE, Jalal Kazempour, Member, IEEE, Pierre Pinson, Senior Member, IEEE, \\ Zacharie De Gréve, Member, IEEE, and François Vallée, Member, IEEE
}

\begin{abstract}
Following a call to foster a transparent and more competitive market, member states of the European transmission system operator are required to publish, among other information, aggregate wind power forecasts. The publication of the latter information is expected to benefit market participants by offering better knowledge of the market operation, leading subsequently to a more competitive energy market. Driven by the above regulation, we consider an equilibrium study to address how public information of aggregate wind power forecasts can potentially affect market results, social welfare as well as the profits of participating power producers. We investigate, therefore, a joint day-ahead energy and reserve auction, where producers offer their conventional power strategically based on a complementarity approach and their wind power at generation cost based on a forecast. In parallel, an iterative game-theoretic approach (diagonalization) is incorporated in order to investigate the existence of an equilibrium for various values of aggregate forecast. As anticipated, variations in public forecasts will affect market results and, more precisely, under-forecasts can mislead power producers to make decisions that favor social welfare, while over-forecasts will cause the opposite effect. Furthermore, energy and reserve market prices can also be affected by deviations in aggregate wind forecasts altering, inevitably, the profits of all power producers.
\end{abstract}

Index Terms-Wind power, aggregate forecasts, public data, equilibrium, game theory.

\section{NOTATION:}

Sets:

$\mathcal{G} \quad$ Set of all conventional power units.

$\mathcal{G}_{\mathcal{J}}$ Set of conventional power units belonging to producer $\mathcal{J}$.

$\mathcal{W} \quad$ Set of all wind power units.

$\mathcal{W}_{\mathcal{J}}$ Set of wind power units belonging to producer $\mathcal{J}$.

$\mathcal{D} \quad$ Set of demands.

$B_{i} \quad$ Set of generation blocks of the $i$-th unit.

$\Omega \quad$ Set of wind power generation scenarios.

$S \quad$ Set of real-time market price scenarios.

The work of L. Exizidis was supported by the public service of WalloniaDepartment of Energy and Sustainable Building, within the framework of the GREDOR project. J. Kazempour and P. Pinson are partly supported by the Danish Strategic Council for Strategic Research through the projects of PROAIN (no. 3045-00012B/DSF) and 5s - Future Electricity Markets (no. 12-132636/DSF).

L. Exizidis, Z. De Gréve and F. Vallée are with the Department of Electrical Engineering, University of Mons, Mons, 7000 Belgium (email: lazaros.exizidis@umons.ac.be; zacharie.degreve@umons.ac.be; francois.vallee@umons.ac.be).

J. Kazempour and P. Pinson are with the Department of Electrical Engineering, Technical University of Denmark, Kgs. Lyngby, 2800 Denmark (e-mail: seykaz@elektro.dtu.dk; ppin@elektro.dtu.dk).
Indices:

$\mathcal{J} \quad$ Index for producers.

$i \quad$ Index for conventional units.

$b \quad$ Index for generation blocks of conventional units.

$d \quad$ Index for demands.

$l \quad$ Index for wind power units.

$\omega \quad$ Index for wind power generation scenarios.

$s \quad$ Index for real-time market price scenarios.

Variables:

$p_{i, b}^{\mathrm{G}} \quad$ Scheduled generation for the $b$-th block of the $i$-th conventional unit [MW].

$r_{i}^{\mathrm{U}} \quad$ Committed upward reserve from the $i$-th conventional unit $[\mathrm{MW}]$.

$r_{i}^{\mathrm{D}} \quad$ Committed downward reserve from the $i$-th conventional unit $[\mathrm{MW}]$.

$\lambda^{\mathrm{DA}} \quad$ Energy price [\$/MWh].

$\mu^{\mathrm{U}} \quad$ Price of capacity for committed upward reserve [\$/MWh].

$\mu^{\mathrm{D}} \quad$ Price of capacity for committed downward reserve [\$/MWh].

$\alpha_{i, b}^{\mathrm{G}} \quad$ Price offer for the $b$-th block of the $i$-th unit [\$/MWh].

$p_{d}^{\mathrm{D}} \quad$ Scheduled consumption for the $d$-th demand [MW].

$p_{l}^{\mathrm{W}} \quad$ Scheduled wind power generation for the $l$-th wind power unit [MW].

$p_{l, \omega}^{\mathrm{W}, \mathrm{RT}}$ Power sold/bought in the real-time market by the $l$-th wind power unit under scenario $\omega[\mathrm{MW}]$.

Parameters:

$C_{i, b}^{\mathrm{G}} \quad$ Marginal cost of the $b$-th block of the $i$-th unit [\$/MWh].

$\lambda_{d}^{\mathrm{D}} \quad$ Price bid of $d$-th demand [\$/MWh].

$\gamma, \delta \quad$ Non-negative factors representing the minimum reserve requirements of the market as percentage of total demand and total installed wind capacity, respectively.

$\bar{W}_{l} \quad$ Installed wind power capacity of the $l$-th unit [MW].

$\bar{P}_{i, b}^{\mathrm{G}} \quad$ Maximum generation capacity of the $b$-th block of the $i$-th unit [MW].

$\bar{P}_{d}^{\mathrm{D}} \quad$ Maximum consumption of the $d$-th demand [MW].

$\bar{R}_{i}^{\mathrm{U}} \quad$ Maximum upward reserve capacity of the $i$-th unit [MW].

$\bar{R}_{i}^{\mathrm{D}} \quad$ Maximum downward reserve capacity of the $i$-th unit [MW]. 
$F_{l} \quad$ Wind power forecast of the $l$-th unit as private data, provided by its owner [MW].

$F^{\mathrm{MO}}$ Aggregate wind power forecast as public information, provided by market operator [MW].

$P_{l, \omega}^{\mathrm{W}, \mathrm{P}}$ Wind power produced by the $l$-th wind power unit under scenario $\omega[\mathrm{MW}]$.

$\lambda_{s}^{\mathrm{RT}} \quad$ Real-time market price under scenario $s$ [\$/MWh].

$\pi_{\omega} \quad$ Probability of scenario $\omega$.

$\pi_{s}^{\lambda} \quad$ Probability of scenario $s$.

\section{INTRODUCTION}

\section{A. Background and Motivation}

$\mathbf{E}$ UROPEAN electricity market regulators, representatives of EU countries and stakeholders gathered in June 2015 in Florence to discuss the implementation of the so-called "third energy package", which aims at improving the functioning of the internal energy markets [1]. Among other, participants agreed on the importance of renewable energy in the energy mix, stressing however the fact that renewable power producers should follow the same rules with conventional producers and compete them without any support, under the current framework of a liberalized market [2]. Moreover, European Union escalated the importance for increased transparency in electricity market operations, which has already improved over the past few years following Regulation $(E U)$ No 543/2013 on the submission and publication of data in electricity markets [3]. The latter implies that transmission system operators (TSOs) shall calculate and provide for their control areas, among other information, a forecast of wind power generation (MW) per bidding zone, per each market time unit of the following day to the European Network of Transmission System Operators (ENTSO-E). Following this directive, TSOs started publishing aggregate forecasts of wind power generation in their control area. For example, Belgium's TSO, i.e., ELIA, publishes day-ahead and weekahead forecasts of wind power [4] in order to "provide the basis for a harmonised, transparent environment and create a level playing field between all market players, which will potentially foster the development of the electricity market" [5]. To this end, ENTSO-E Transparency Platform [6] provides free, continuous access to Pan-European electricity market data for all users, across six main categories: load, generation, transmission, balancing, outages and congestion management. However, Europe is not alone in taking decisive steps towards public forecast-related information. The interest for public aggregate forecasts is shared by more system operators in other geographical areas, such as the Independent System Operator of New England [7], Midcontinent Independent System Operator [8] and Alberta Electric System Operator [9], which provide aggregate day-ahead and/or week-ahead forecasts for their control areas.

Despite the aforementioned political decisions and policy regulations, according to the authors' knowledge, there is yet no technical paper in the power systems literature that studies the impact of public aggregate forecasts on market operation and market participants' interests. Therefore, the main contribution of this paper is to offer an insight into how public forecasts and the level of their deviation can potentially impact market operation as well as market participants' interests. The study is performed for a day-ahead (DA) market setup with increased share of wind power. A joint energy and reserve auction is considered, where strategic conventional power producers may also include within their offering portfolio wind power, which they offer at generation cost based on a forecast. Given that producers may exercise market power, it comes naturally to represent the market by a non-cooperative game played among all producers. We consider all producers as strategic players, given that an agentbased approach would not allow us to incorporate the fact that all producers have access to the same information and, thus, they all have the capacity to exercise market power. Therefore, a game-theoretic approach is followed where each producer builds a mathematical program with equilibrium constraints (MPEC) in order to optimally decide its offering strategy. An iterative diagonalization approach [10]-[13] is then incorporated to search for the equilibrium of the game, which is found when no producer has incentive to change its strategy unilaterally.

\section{B. Literature Review and Contributions}

The major drawback for the large-scale integration of wind power in electricity markets is its intermittent nature. The cost for backup flexibility reserves is considerably high in order to guarantee reliability, while energy storage is still not mature enough [14]. Despite the fact that wind power forecasting will never be perfectly accurate, it has improved significantly through intensive research spanning over the last two decades [15]. Following the emergence of advanced methods for wind power forecasting, the latter has been distinguished as a dominant tool for market participation. Under this context, an ever-increasing number of contributions is focusing on suggesting tools for wind power trading in electricity markets with significant share of wind power generation.

Initial studies adopted models where wind power producers are non-strategic players, i.e., price-takers, and/or receiving additional support when participating in a forward electricity market [16]-[19]. However, as the cost of wind power production is low and the competitiveness of wind power increases, wind power producers are forced to participate in the electricity markets under full competition and following the same rules as conventional producers [3]. Under this context, [20] considers that wind power producers strategically offer their power in the balancing market. It is anticipated that wind producer acts as a price-taker in DA market, due to the large volume of traded energy. In addition, [20] investigates how the shape of the forecast distribution impacts the offering strategy of producer. The problem of a price-maker wind power producer in DA market, being a deviator in the balancing market, is addressed in [21]. More specifically, the problem is formulated as a stochastic optimization tool for market participation, where uncertainty pertaining to wind power production is represented through scenarios. The impact of a price-maker wind power producer on electricity prices as well as on the resulting imbalances is studied in [22] for 
a market without regulated tariffs. Furthermore, study [23] additionally considered, through scenarios, the uncertainties in demand, wind power generation and bidding strategies of strategic conventional generators focusing on the problem of strategic wind power trading.

Since strategies of rival traders in an electricity market are a priori not known, a more realistic setup would suggest the consideration of multiple strategic power producers competing each other in an effort to increase their own profits. Considering various market players that offer their generation strategically acting as price-makers, the investigated market can be seen as a non-cooperative game assuming complete information knowledge. For example, in [24], the equilibria reached by strategic producers in a pool-based electricity market are investigated. The behavior of each power producer is described by an MPEC, whose joint solution constitutes an equilibrium program with equilibrium constraints (EPEC). Moreover, in [25], strategic electricity producers react to both prices and rival production changes, in both the spot and the futures markets. The proposed model allows deriving analytical expressions that characterize such multi-market equilibria. Motivated by the increasing levels of wind power penetration in electricity markets, [26] investigates the equilibria in a pool-based oligopolistic electricity market, including a DA and several real-time (RT) markets, where wind power is also considered within the generation portfolio of the strategic producers. Then, the resulting EPEC is solved, in the search of the equilibrium point. Following the same approach, [27] proposes a stochastic model to find the equilibria that, compared to [26], additionally considers the transmission constraints and proposes a different approach for RT market clearing.

In view of the above, the central contribution of this paper is to investigate the impact of public aggregate wind forecasts in a competitive market environment. Even though availability of public aggregate wind forecasts is expected to improve market operation, strategic behaviour of market players could merely jeopardize this vision. More precisely, the impact of aggregate forecasts on the market results should be investigated, given a market with strategic players. Therefore, we study a market setup where producers, which include within their portfolio both wind and conventional power units, offer their conventional power strategically to the DA market, while their wind power is offered at generation cost based on a forecast. Producers determine their optimal offering strategy considering individual forecasts for their own wind units as well as the public aggregate forecast. Their decision-making tool is formulated as a bilevel optimization model. Then, the interaction of all participating producers is represented as a non-cooperative game with complete information, whose equilibrium is investigated through an iterative diagonalization procedure. Energy and reserve prices as well as social welfare are compared for under- and over-forecasting at the equilibrium points. Additionally, the results are analyzed from the producers' point of view by evaluating the impact of different aggregate forecasts on their profits. Note that the contributions of this study are not methodological, in the sense that we do not present a new decision-making model or methodology for finding the equilibria. On the contrary, we build a decision- making tool based on available models in literature, and investigate the new act of European electricity markets, i.e., publishing aggregate wind forecasts, and its impact on market outcomes.

\section{Paper Organization}

The rest of the paper is organized as follows: Section II proposes a bilevel optimization model for the strategic offering of power producers and provides the corresponding mathematical formulation. Additionally, it presents the methodology followed for the equilibrium study among the various power producers. Section III presents the results for a case-study based on the IEEE reliability test system, as well as an additional numerical study considering uncertainty of wind generation and RT prices. Finally, Section IV concludes the paper.

\section{MODEL}

This section is divided into four parts: Section II-A presents the main features and assumptions of the model used in this paper. Section II-B provides the mathematical formulation of producers' decision-making tool, while the iterative diagonalization process followed to identify the equilibrium of the game is described in Section II-C. Lastly, an additional step is taken in Section II-D, where the actual DA market is cleared based on the offers of producers at the equilibrium point.

\section{A. Features and Assumptions}

An imperfectly competitive electricity market is considered, in which power producers include within their generation portfolio both conventional and wind power generation. Producers behave strategically with respect to price offers of conventional generation [26], [28], but not regarding their wind generation, which they offer deterministically based on a forecast. For the sake of simplicity, transmission constraints are not enforced [20], [22], [26]. In addition, the inter-temporal constraints, e.g., ramping limits of conventional units, are not enforced and thus a single-hour auction is considered. This is consistent with the relevant literature [20], [23], [24], [26]. In this study, we only focus on DA market and evaluate its impact on market outcomes in that stage. The RT stage, in which the actual wind power is realized, is not considered in this study. Market operator commits in DA a specific level of reserve capacity, which manages the potential DA forecast errors in real time. The required reserve capacity is exogenously sized based on the total demand as well as on the installed wind capacity. However, a complementary numerical study is additionally presented in Section III-D, offering an insight on the same problem considering uncertainty on wind power generation and RT prices. Finally, we assume that the wind production cost is zero and demand is deterministic and elastic with respect to price.

\section{B. Bilevel Model for Each Producer}

In this subsection, the mathematical formulation of the producers' offering tool is presented. Each producer solves bilevel problem (1) in order to optimize its offer, considering its privately owned wind power forecast as well as 
the available public aggregate wind power forecast. The problem is formulated as a bilevel model for each producer $\mathcal{J}$, whose upper-level, i.e., (1a)-(1c), maximizes producer's profit and derives its strategic price offers and whose lowerlevel problem, i.e., (1d)-(1o), clears the DA market through maximizing social welfare. The upper-level objective function (1a) is constrained by both upper-level constraints (1b)-(1c) and lower-level problem (1d)-(1o). Note that dual variables are indicated in each lower-level constraint after a colon.

$$
\begin{aligned}
& \left\{\underset{\alpha_{i, b}^{\mathrm{G}}, \Xi^{\mathrm{LL}, \mathrm{P}} \cup \Xi^{\mathrm{LL}, \mathrm{D}}}{\operatorname{Maximize}} \sum_{i \in \mathcal{G}_{\mathcal{J}}} \sum_{b \in B_{i}}\left(\lambda^{\mathrm{DA}}-C_{i, b}^{\mathrm{G}}\right) p_{i, b}^{\mathrm{G}}\right. \\
& +\sum_{l \in \mathcal{W}_{\mathcal{J}}}\left(\lambda^{\mathrm{DA}} p_{l}^{\mathrm{W}}\right)+\sum_{i \in \mathcal{G}_{\mathcal{J}}}\left(\mu^{\mathrm{U}} r_{i}^{\mathrm{U}}+\mu^{\mathrm{D}} r_{i}^{\mathrm{D}}\right) \\
& \underset{\Xi \text { LL,P }}{\operatorname{maximize}} \sum_{d \in \mathcal{D}} \lambda_{d}^{\mathrm{D}} p_{d}^{\mathrm{D}}-\sum_{i \in \mathcal{G}} \sum_{b \in B_{i}} \alpha_{i, b}^{\mathrm{G}} p_{i, b}^{\mathrm{G}}
\end{aligned}
$$

where $\Xi^{\mathrm{LL}, \mathrm{P}}=\left\{p_{l}^{\mathrm{W}}, p_{i, b}^{\mathrm{G}}, p_{d}^{\mathrm{D}}, r_{i}^{\mathrm{U}}, r_{i}^{\mathrm{D}}\right\}$ is the set of primal variables of lower-level problem (1d)-(1o). Furthermore, $\Xi^{\text {LL,D }}=$ $\left\{\lambda^{\mathrm{DA}}, \mu^{\mathrm{U}}, \mu^{\mathrm{D}}, \underline{\rho}_{i}^{\mathrm{U}}, \bar{\rho}_{i}^{\mathrm{U}}, \underline{\rho}_{i}^{\mathrm{D}}, \bar{\rho}_{i}^{\mathrm{D}}, \bar{\tau}_{i, b}, \underline{\tau}_{i, b}, \underline{\phi}_{i}^{\mathrm{G}}, \bar{\phi}_{i}^{\mathrm{G}}, \underline{\psi}_{d}, \bar{\psi}_{d}\right.$, $\left.\underline{\sigma}_{l}, \bar{\sigma}_{l}, \underline{\sigma}^{\mathrm{MO}}, \bar{\sigma}^{\overline{\mathrm{MO}}}\right\}$ is the set of dual variables of the lowerlevel problem. Finally, the primal variables of the upper-level problem (1a)-(1c) are $\alpha_{i, b}^{\mathrm{G}}$ as well as all members of variable sets $\Xi^{\mathrm{LL}, \mathrm{P}}$ and $\Xi^{\mathrm{LL}, \mathrm{D}}$.
The upper-level objective function, i.e., (1a), maximizes profit of producer $\mathcal{J}$, and includes:

- Producer's profit due to conventional generation after deducting the generation costs, i.e., $\sum_{i \in \mathcal{G}_{\mathcal{J}}} \sum_{b \in B_{i}}\left(\lambda^{\mathrm{DA}}-\right.$ $\left.C_{i, b}^{\mathrm{G}}\right) p_{i, b}^{\mathrm{G}}$.

- Producer's profit due to wind generation, i.e., $\sum_{l \in \mathcal{W}_{\mathcal{J}}}\left(\lambda^{\mathrm{DA}} p_{l}^{\mathrm{W}}\right)$.

- Associated profits for providing upward and downward reserve capacities, i.e., $\sum_{i \in \mathcal{G}_{\mathcal{J}}}\left(\mu^{\mathrm{U}} r_{i}^{\mathrm{U}}+\mu^{\mathrm{D}} r_{i}^{\mathrm{D}}\right)$.

The upper-level constraints (1b)-(1c) impose the strategic price offer for the conventional units, i.e., $\alpha_{i, b}^{\mathrm{G}}$, to be nonnegative and non-decreasing from the first offer block to the last. The lower-level objective function (1d) maximizes social welfare of the market based on producers' offers and demands' bids, which is constrained by (1e)-(1o). Constraint (1e) enforces the power balance in DA. Constraints (1f) and $(1 \mathrm{~g})$ impose the minimum reserve requirements of the market. The level of minimum reserve requirements is introduced as a portion of the total load level plus a portion of the level of installed wind power capacity, using non-negative factors $\gamma$ and $\delta$, respectively. Note that the dual variables of constraints (1e), (1f) and (1g) indicate the prices for energy, upward and downward committed reserves, respectively. Generation and reserve capacities are imposed by constraints (1h)-(11) while the scheduled demand should be lower than or equal to the total demand, imposed by (1m). Constraint (1n) sets the scheduled wind for the investigated producer to be less than or equal to its own private forecast. Finally, the aggregate scheduled wind power of rival wind units should be lower than or equal to the public aggregate forecast minus producer's own individual forecast, enforced by (10).

Bilevel (1) is transformed into an MPEC, which is presented in Appendix A. The corresponding MPEC is linearized and then solved, as a mixed-integer linear programming problem. The collection of all MPECs, one per producer, forms an EPEC, whose solution identifies the equilibrium point. Unlike [24]-[26] in which the lower-level problem of all producers is common (equilibrium with shared constraints), the lowerlevel problem of producers in this paper is different. This prevents the use of EPEC solution techniques proposed in [24]-[26]. Alternatively, we use an iterative diagonalization approach to solve EPEC, in which each producer determines sequentially its strategy considering the rivals' strategies fixed. The iterations continue until no producer changes its strategy unilaterally or until the maximum number of iterations is reached. Further description is available in the following section.

\section{Searching the Equilibrium Point Among Producers}

We represent the game among all strategic producers by an iterative diagonalization process, which is illustrated in Fig. 1. Producers make their offering decisions sequentially, while at each step each producer considers the offers of rivals being fixed. The game is, therefore, described by the following three steps:

1) Iteration counter $(c)$, maximum number of iterations $\left(c_{\max }\right)$, convergence tolerance $(\varepsilon)$ and price offering 


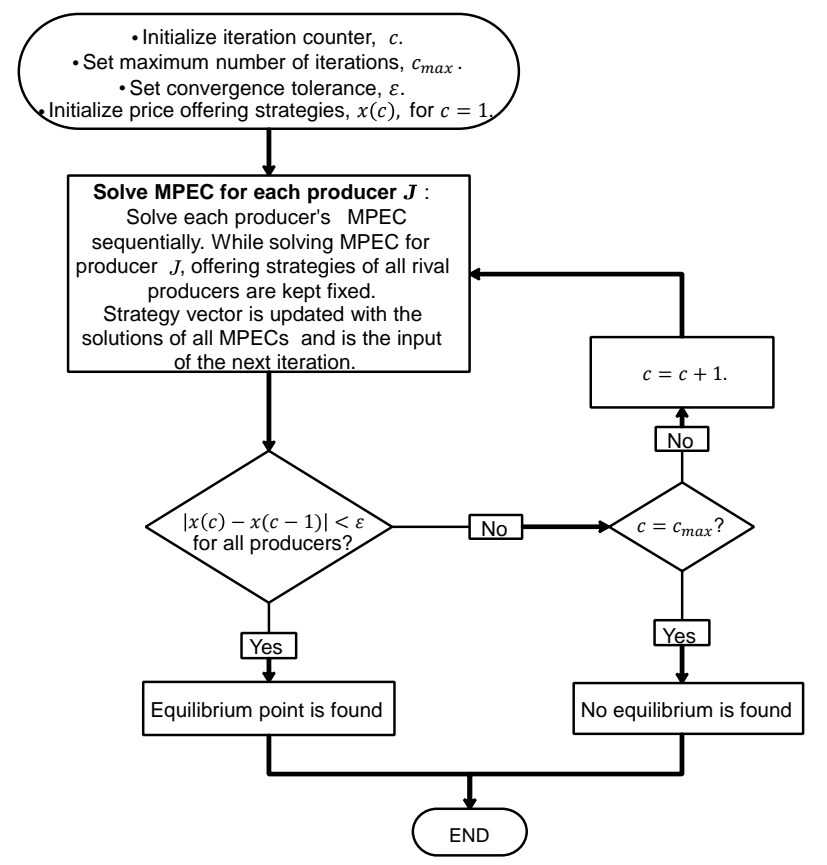

Fig. 1. The iterative diagonalization approach to identify the equilibrium point.

strategies $(x(c))$ are initialized. For the first step of the iterative process, producers' price offering strategies, i.e., vector of price offers, are initialized to be equal to their marginal costs.

2) Through iteration $c$, each power producer solves the corresponding MPEC in order to determine its optimal offering strategy, keeping rivals' offers fixed to their value at iteration $c-1$. The vector of price offers is, thus, updated by the solution of the corresponding MPEC for all producers.

3) Finally, vector of price offers at iteration $c$ is compared to the one at iteration $c-1$. If their mathematical distance is greater than $\varepsilon$, then a new iteration starts. The iterations stop either if this distance is smaller than the value of tolerance, i.e., an equilibrium is found, or if the maximum number of iterations has been reached, indicating that no equilibrium is found.

\section{Day-ahead Market Clearing}

The answer to the central question of this work, i.e., investigating the impact of public aggregate wind forecast on market results, requires taking an additional step where DA market is actually cleared by the market operator based on producers' offers and demands' bids. Note that each producer subjectively anticipates DA market clearing, taking into consideration the aggregate wind power forecast. However, the market is actually cleared by the market operator based solely on producers' wind power offers, which are equal to their private wind power forecast. Price offers for conventional generation at the equilibrium point, derived by the models presented in Sections II-B and II-C, are also considered for the market clearing. Therefore, the formulation of the DA market is given by (2) below:
TABLE I

Technical Characteristics of Power Generation Units [MW]

\begin{tabular}{|c|c|c|c|c|c|c|c|}
\hline $\begin{array}{l}\text { Producer } \mathcal{J} \text { with } \\
\text { generation port- } \\
\text { folio } i \text { and } l\end{array}$ & $\bar{P}_{i, b 1}^{\mathrm{G}}$ & $\bar{P}_{i, b 2}^{\mathrm{G}}$ & $\bar{P}_{i, b 3}^{\mathrm{G}}$ & $\bar{P}_{i, b 4}^{\mathrm{G}}$ & $\bar{R}_{i}^{\mathrm{D}}$ & $\bar{R}_{i}^{\mathrm{U}}$ & $\begin{array}{l}\text { Wind power } \\
\text { forecast by } \\
\text { producer }\left(F_{l}\right)\end{array}$ \\
\hline$j 1$ & 60.8 & 91.2 & 91.2 & 60.8 & 80 & 80 & 200 \\
\hline$j 2$ & 75 & 75 & 90 & 60 & 75 & 75 & 350 \\
\hline$j 3$ & 206.85 & 147.75 & 118.2 & 118.2 & 120 & 120 & 450 \\
\hline$j 4$ & 12 & 18 & 18 & 12 & 0 & 0 & 400 \\
\hline$j 5$ & 217 & 155 & 124 & 124 & 180 & 180 & - \\
\hline$j 6$ & 200 & 200 & 240 & 160 & 80 & 80 & - \\
\hline$j 7$ & 300 & 0 & 0 & 0 & 0 & 0 & - \\
\hline$j 8$ & 140 & 87.5 & 52.5 & 70 & 80 & 80 & \\
\hline
\end{tabular}

$$
\underset{p_{i, b}^{\mathrm{G}}, p_{l}^{\mathrm{W}}, p_{d}^{\mathrm{D}}, r_{i}^{\mathrm{U}}, r_{i}^{\mathrm{D}}}{\operatorname{Maximize}} \sum_{d \in \mathcal{D}} \lambda_{d}^{\mathrm{D}} p_{d}^{\mathrm{D}}-\sum_{i \in \mathcal{G}} \sum_{b \in B_{i}} \alpha_{i, b}^{\mathrm{G}} p_{i, b}^{\mathrm{G}}
$$

subject to:

$$
\begin{aligned}
& (1 \mathrm{e})-(1 \mathrm{~m}) \\
& 0 \leq p_{l}^{\mathrm{W}} \leq F_{l} \quad \forall l .
\end{aligned}
$$

Note that the results obtained from lower-level problem in model (1) and those obtained from model (2) are not necessarily the same, though their optimization structure is similar. In the lower-level problem of model (1) corresponding to a particular producer, the market is cleared from the producer's perspective based on the available data for that producer, i.e., forecast for its own wind power units and public aggregate wind forecast. However, model (2) actually clears the market from market operator's point of view considering the offer prices received from (1) and the private wind forecasts of producers. Therefore, constraints $(2 \mathrm{~b})$ are the same with lowerlevel problem $(1 \mathrm{e})-(1 \mathrm{~m})$ of each producer but lower-level constraints (1n) and (1o) are now replaced by (2c) for all producers. In addition $\alpha_{i, b}^{\mathrm{G}}, \forall i, \forall b$ are now parameters, derived from the equilibrium model presented in Section II-C.

\section{A. Data}

\section{CASe Study}

A single-hour case-study based on the IEEE one-area reliability test system [29] is considered. For the sake of simplicity, conventional units are grouped by type. Each conventional unit offers at a quantity identical to its installed capacity, given in Table I, and at a strategic price that can differ from its marginal costs, which are presented in Table II. Among eight producers competing in this case study, four of them, namely producers $j 1, j 2, j 3$ and $j 4$, own wind power units in addition to their conventional generation capacity. Each of those producers forecasts its own wind power generation deterministically. The forecasted values are listed in the last column of Table I. Note that these individual forecasts are private data and are not shared with the rivals. The demand levels and the corresponding bids are presented in Table III while the factors defining the minimum reserve requirements are arbitrarily chosen as $\gamma=0.05$ and $\delta=0.1$. Finally, the computational issues are discussed in Appendix C.

\section{B. Reference Case: Public Aggregate Wind Forecast Equals the Sum of Private Individual Forecasts}

In real-world markets, producers with wind power within their generation portfolio usually make their offers to the 
TABLE II

Marginal Costs of Conventional Units [\$/MWh]

\begin{tabular}{c|c|c|c|c} 
& $C_{i, b 1}^{\mathrm{G}}$ & $C_{i, b 2}^{\mathrm{G}}$ & $C_{i, b 3}^{\mathrm{G}}$ & $C_{i, b 4}^{\mathrm{G}}$ \\
\hline$j 1$ & 11.46 & 11.96 & 21.67 & 22.72 \\
$j 2$ & 18.60 & 20.03 & 21.67 & 22.72 \\
$j 3$ & 19.20 & 20.32 & 21.22 & 22.13 \\
$j 4$ & 23.41 & 23.78 & 26.84 & 30.4 \\
$j 5$ & 9.92 & 10.25 & 10.68 & 11.26 \\
$j 6$ & 5.31 & 5.38 & 5.53 & 5.66 \\
$j 7$ & 2.00 & - & - & - \\
$j 8$ & 10.08 & 10.66 & 11.09 & 11.72 \\
\hline
\end{tabular}

TABLE III

DEMAND CHARACTERISTICS

\begin{tabular}{l|c|c} 
& Demand level $[\mathrm{MW}]$ & Demand bid [\$/MWh] \\
\hline$d 1$ & 550 & 65 \\
$d 2$ & 300 & 60 \\
$d 3$ & 500 & 55 \\
$d 4$ & 300 & 55 \\
$d 5$ & 200 & 52 \\
$d 6$ & 450 & 52 \\
$d 7$ & 500 & 50 \\
$d 8$ & 200 & 50 \\
$d 9$ & 300 & 50 \\
$d 10$ & 200 & 50 \\
\hline Total & 3500 & - \\
\hline
\end{tabular}

market based on a deterministic forecast, which is privately generated and accessible. Undoubtedly, the sum of these individual forecasts differs from the aggregate forecast of the market operator. A special case, according to which the sum of private individual wind forecasts equals the public aggregate one, is used throughout this study as a reference. In this case, the public aggregate wind forecast is $1400 \mathrm{MW}$, which is the sum of individual wind forecasts reported in the last column of Table I.

For the reference case, the strategic price offers of producers are derived and presented in Table IV. Producers $j 1, j 2, j 5, j 6$ and $j 8$ offer their cheap generation blocks at zero price in order to get scheduled. The energy price is $\$ 18.601 / \mathrm{MWh}$ as presented in Table V. However, the corresponding capacity prices for committed downward and upward reserve are both zero. This is explained by the last two columns of Table VI, where it is observed that producers $j 1, j 2$ and $j 3, j 8$ have still additional available downward and upward reserve capacity, respectively. Therefore, constraints (1f) and (1g), which enforce the minimum reserve requirements of the market, are not binding and the corresponding dual variables are zero. Table VI additionally presents producers' total profits for the reference case, as well as the scheduled power for each generation block of each producer. Note that each wind farm is scheduled in DA market at a quantity equal to its owner's wind power forecast.

\section{Public Aggregate Wind Forecast Differs from the Sum of Private Individual Forecasts}

In this section we investigate the impact of deviations between public aggregate wind forecast and the sum of private ones, on market results and on producers' profits. For this purpose we search the equilibrium under different values of aggregate forecast, which may not be equal to the one corresponding to the reference case. The problem is solved for aggregate forecast that takes values in a wide range
TABLE IV

Strategic Price OfFers at THE EQUilibrium PoINT FOR REFERENCE CASE $[\$ / M W h]$

\begin{tabular}{c|c|c|c|c} 
& $\alpha_{i, b 1}^{\mathrm{G}}$ & $\alpha_{i, b 2}^{\mathrm{G}}$ & $\alpha_{i, b 3}^{\mathrm{G}}$ & $\alpha_{i, b 4}^{\mathrm{G}}$ \\
\hline$j 1$ & 0 & 18.600 & 18.601 & 18.602 \\
$j 2$ & 0 & 18.601 & 18.602 & 18.603 \\
$j 3$ & 18.601 & 18.602 & 18.603 & 18.604 \\
$j 4$ & 18.601 & 18.602 & 18.603 & 18.604 \\
$j 5$ & 0 & 0.001 & 0.002 & 18.600 \\
$j 6$ & 0 & 0.001 & 0.002 & 18.601 \\
$j 7$ & 18.600 & - & - & - \\
$j 8$ & 0 & 0.001 & 0.002 & 18.601 \\
\hline
\end{tabular}

TABLE V

Energy and Reserve Prices for Reference Case

\begin{tabular}{c|c} 
& Price [\$/MWh] \\
\hline$\mu^{\mathrm{U}}$ & 0 \\
$\mu^{\mathrm{D}}$ & 0 \\
$\lambda^{\mathrm{DA}}$ & 18.601 \\
\hline
\end{tabular}

around the reference case, which is used for comparison. More specifically, aggregate forecast is considered to take different values between $900 \mathrm{MW}$ and $1900 \mathrm{MW}$ in 10-MW steps. Recall that the sum of private forecasts is $1400 \mathrm{MW}$ (reference case). Note that for values of aggregate forecast that no equilibrium is found, there are no results to be presented.

Fig. 2 shows energy and reserve prices at the equilibrium point for different aggregate wind forecast values. It is observed that energy price is zero for low values of aggregate forecast, i.e., below $1030 \mathrm{MW}$. Then, following some small fluctuations, it stabilizes at $\$ 18.601 / \mathrm{MWh}$. Zero energy price for small values of aggregate wind forecast is explained by producers' price offers. More particularly, when the aggregate wind forecast is low, the producers expect low wind power penetration in the market based on their decision-making model (1). The producers do not have information on rivals' wind offers and therefore anticipate market outcomes based on the available aggregate wind forecast. Thus, from producers' perspective, the expected low wind power penetration (low aggregate forecast) indicates potentially high market-clearing price. This motivates some producers to offer at zero price in order to get scheduled. However, this has the opposite result in terms of actual market outcomes. Producers' zero price offers along with the total wind power (which is higher than producers anticipated) lead to zero market-clearing price. Moreover, prices for committed downward reserve are always zero in this study, which is explained by the fact that there is sufficient capacity for downward reserve, without incurring extra cost. In other words, the corresponding reserve requirement never changes the power schedule of generators. However, prices for committed upward reserve can take non-zero values, especially for values of aggregate forecast below and around $1400 \mathrm{MW}$. From Fig. 3 it is observed that for small values of aggregate forecast, profits of all producers are either zero or negative. This result is again explained by the producers' expectations for high energy prices, which inevitably leads to the opposite results, i.e., zero prices, with the consequent impact on producers' profits. It should be noted that the producers' offers are derived from model (1) and are based on their available information. Accordingly, producers do not anticipate their 
TABLE VI

SCHedules And Producers' Profits at THE EQuilibrium POINT FOR THE REFERENCE CASE

\begin{tabular}{l||l||l|l|l} 
& Profit [\$] & $\begin{array}{l}\text { Scheduled power per gen- } \\
\text { eration block [MW] }\end{array}$ & $\begin{array}{l}\text { Committed capac- } \\
\text { ity for upward re- } \\
\text { serve [MW] }\end{array}$ & $\begin{array}{l}\text { Committed capac- } \\
\text { ity for downward } \\
\text { reserve [MW] }\end{array}$ \\
\hline$j 1$ & 4760 & $60.8 ; 91.2 ; 0 ; 0$ & 80 & 0 \\
$j 2$ & 6510 & $75.0 ; 0 ; 0 ; 0$ & 75 & 35 \\
$j 3$ & 8370 & $0 ; 0 ; 0 ; 0$ & 70 & 0 \\
$j 4$ & 7382 & $12.0 ; 0 ; 0 ; 0$ & 0 & 0 \\
$j 5$ & 5070 & $217.0 ; 155.0 ; 124.0 ; 124.0$ & 0 & 180 \\
$j 6$ & 8711 & $200.0 ; 200.0 ; 240.0 ; 21.0$ & 80 & 80 \\
$j 7$ & 4980 & $300.0 ; 0 ; 0 ; 0$ & 0 & 0 \\
$j 8$ & 2282 & $140.0 ; 87.5 ; 52.5 ; 0$ & 70 & 80 \\
\hline
\end{tabular}

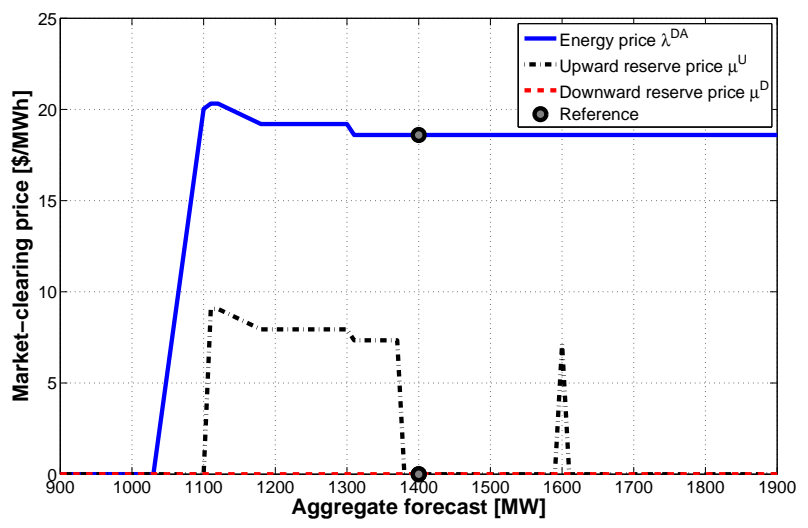

Fig. 2. Energy and reserve prices for different aggregate forecast values

profits to be negative. For example, Fig. 4 shows that, apart from producer $j 7$ (red bars) who is not scheduled for aggregate forecasts below $1030 \mathrm{MW}$, all other producers are scheduled in the DA market. As already discussed, in the case of small aggregate forecasts, producers mostly offer their generation at zero price in order to get scheduled, expecting that the energy price will actually be high. However, energy price is eventually zero, leading to negative profits for producers. Obviously, this is an unfavorable result for the producers, which happened due to their decision-making process that depends on public aggregate forecasts. This result can be better understood by comparing models (1) and (2) of Sections II.B and II.D, respectively. Producers anticipate market outcomes based on model (1) that considers the public aggregate forecast, i.e., constraint (1o), while the actual market is cleared based on wind offers, which are fixed and equal to the individual wind forecasts of producers, i.e., constraint (2c).

In addition, social welfare is also affected by the level of aggregate forecast. In Fig. 5, we present the social welfare calculated as the value obtained for objective function (2a). Social welfare is not directly affected by public aggregate forecast - which has a direct impact on the producers decisionmaking model (1). In turn, producers' strategic decisions, i.e., price offers of conventional generation, have a direct impact on the market-clearing results and, thus, on social welfare. Even though producers always offer their wind power generation based solely on their private wind forecast, their price offers for conventional generation are highly affected by the level of

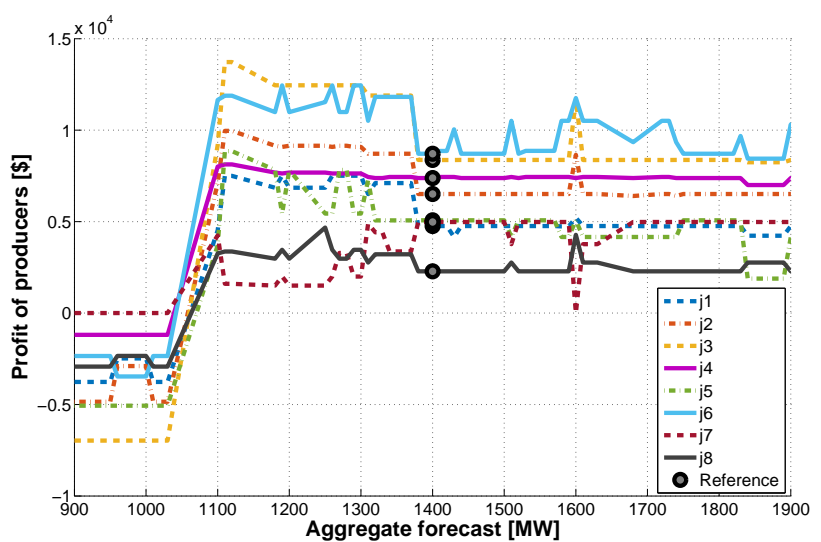

Fig. 3. Producers' profits for different aggregate forecast values

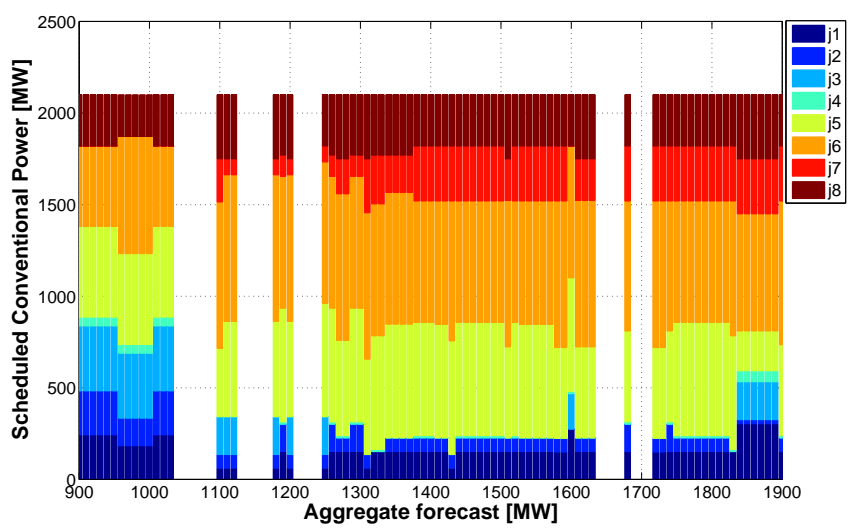

Fig. 4. Day-ahead schedules of conventional generators for different aggregate forecast values

public aggregate forecast, with consequent impact on marketclearing outcomes and social welfare. In line with the previous description, low values of aggregate forecast lead to low price offers from producers and, therefore, cheaper energy is scheduled in the market. Consequently, social welfare is increased for low aggregate wind forecast. Likewise, higher values of aggregate forecast lead to lower social welfare, caused by comparatively high price offers.

For further clarity, the supply-demand curves for some cases of specific interest are presented in Fig. 6. As described earlier, the wind power offers are always equal to the producers' individual forecasts at zero price. However, price offers for conventional generators are different, derived from model (1), which depends on the level of aggregate wind forecast. Hence, one can see that the price offers at $F^{\mathrm{MO}}=1030 \mathrm{MW}$ lead to zero energy price while in cases of $F^{\mathrm{MO}}=1400 \mathrm{MW}$ and $F^{\mathrm{MO}}=1500 \mathrm{MW}$ energy price is $\$ 18.601 / \mathrm{MWh}$. The case of $F^{\mathrm{MO}}=1200 \mathrm{MW}$ is of specific interest given that the upward reserve constraint is active. Therefore, even though the supplydemand curve sets the energy price at $\$ 11.26 / \mathrm{MWh}$ (see point in circle in Fig. 6), the actual energy price derived from model (2) is $\$ 19.20 / \mathrm{MWh}$, due to the positive price for the upward reserve. 


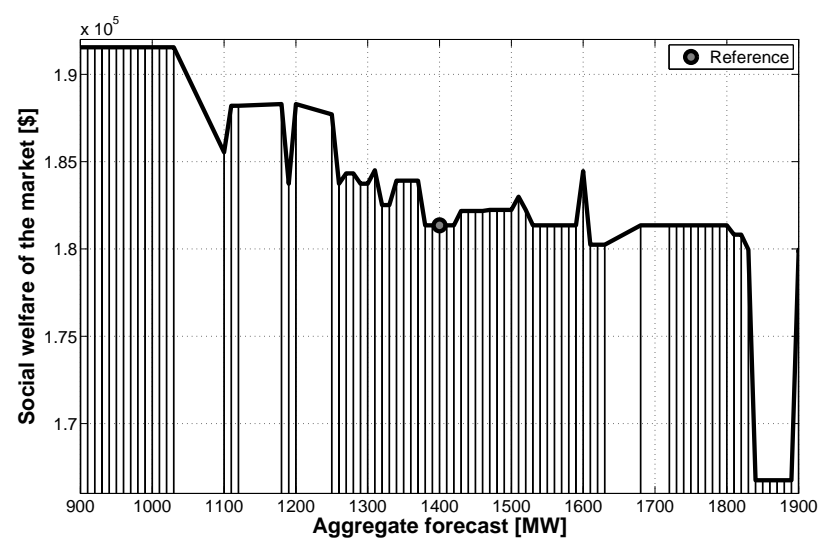

Fig. 5. Social welfare of the market for different aggregate forecast values

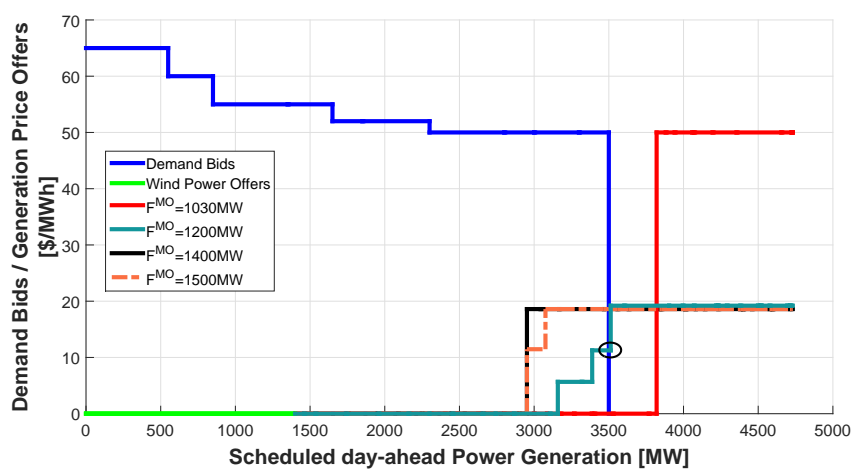

Fig. 6. Supply-demand curves for different aggregate forecast values

\section{Uncertainty of Wind Forecasts and Real-Time Prices}

In the previous sections we have focused on a DA market setup only. However, it is of further interest to confirm the validity of the aforementioned results based on a case-study accommodating a certain degree of uncertainty stemming from the RT market stage. Indeed, considering the uncertainty of wind power generation along with the anticipation of the RT market prices can enable the derivation of advanced strategies for wind power producers, avoiding additional costs due to forecast errors in the RT stage. Using as a basis the presented case-study, we search the equilibrium of the market under a number of scenarios for the RT wind power generation as well as the RT prices, following the approach of [21]. According to the above, model (1) is transformed into the new problem (4), the formulation of which is presented in Appendix B. The input data for this numerical case-study are presented in Tables VII and VIII, defining three scenarios for each source of uncertainty. Note that the deterministic forecast used in the previous sections, is equal to the expected values of the three wind scenarios. Furthermore, similarly to [21], we assume that wind power producers are price-takers in the RT market and, thus, they do not affect RT prices.

Following the same presentation as before, we present the two most interesting figures, i.e, social welfare and producers' profits for various levels of aggregate forecasts. In Fig. 7, a similar trend with the analysis of Section III-C is observed. Social welfare is comparatively lower for greater values of
TABLE VII SCENARios For Wind POWER GENERATION $\left(P_{l, \omega}^{\mathrm{W}, \mathrm{P}}\right)[\mathrm{MW}]$

\begin{tabular}{l|l|l|l} 
& $\omega_{1}$ & $\omega_{2}$ & $\omega_{3}$ \\
\hline \hline$\pi_{\omega}$ & 0.2 & 0.3 & 0.5 \\
\hline$j 1$ & 160 & 250 & 186 \\
\hline$j 2$ & 260 & 320 & 404 \\
\hline$j 3$ & 400 & 430 & 482 \\
\hline$j 4$ & 320 & 380 & 444 \\
\hline$j 5$ & - & - & - \\
\hline$j 6$ & - & - & - \\
\hline$j 7$ & - & - & - \\
\hline$j 8$ & - & - & - \\
\hline
\end{tabular}

TABLE VIII

SCEnARIOS FOR REAL-TIME Prices [ $\$ / M W h]$

\begin{tabular}{l|l|l|l} 
& $s_{1}$ & $s_{2}$ & $s_{3}$ \\
\hline \hline$\pi_{s}^{\lambda}$ & $\frac{1}{3}$ & $\frac{1}{3}$ & $\frac{1}{3}$ \\
\hline \hline$\lambda_{s}^{\mathrm{RT}}$ & 10 & 20 & 30 \\
\hline
\end{tabular}

aggregate forecasts. However, the possibility of having high RT prices may weaken the aforementioned decreasing profile of social welfare. Finally, Fig. 8 is similar to Fig. 3 of the DAonly study, where it is observed that for very small values of aggregate forecasts producers may earn zero or even negative profits. The interpretation of this outcome is already presented in detail in Section III-C.

\section{Conclusions And Prospects}

An increasing interest towards transparency and competitiveness in energy markets has led to decisions and directives for the publication of various market-related information. To this end, system operators invest in generating and publishing qualitative market data, including aggregate wind power forecast, envisioning an improved and transparent market operation. In the presence of a public aggregate wind forecast, participating producers may consider this information in their decision-making tool. This paper uses a complementarity model that provides producers with the strategic price offers for their conventional generation, while wind power is offered at zero price based on an individual forecast. The main scope of the study is, by using the aforementioned model, to investigate how the availability of public aggregate forecasts can affect electricity market outcomes. To this end, we considered multiple strategic producers and described the market as a noncooperative game, whose equilibrium is approached through an iterative diagonalization technique.

The results of this equilibrium study indicate that marketclearing prices (energy and reserve) and social welfare of the market could be significantly affected by the public aggregate wind forecast. More precisely, the under-forecast of aggregate wind power leads to comparatively low or even zero energy prices. Producers expect high prices due to decreased wind power penetration and thus make low price offers for a portion of their generation portfolio in order to get scheduled. Low price offers inevitably impact social welfare, which increases accordingly. The opposite effect is observed for 


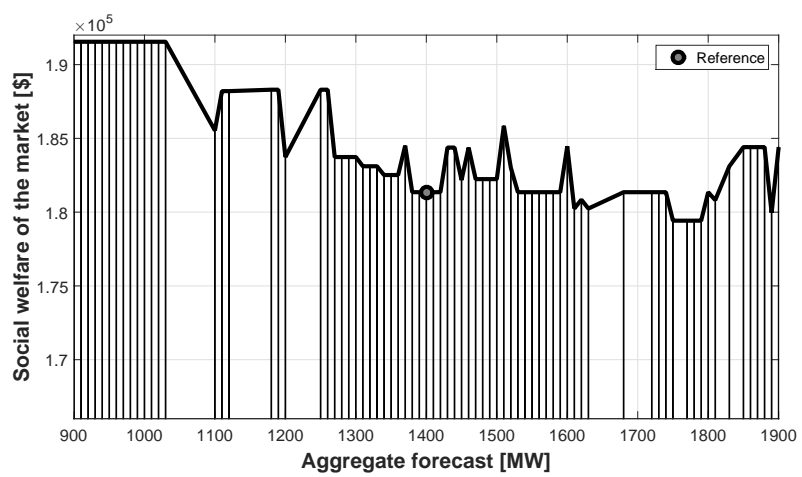

Fig. 7. Social welfare of the market for different aggregate forecast values considering uncertainty in wind power generation and real-time prices

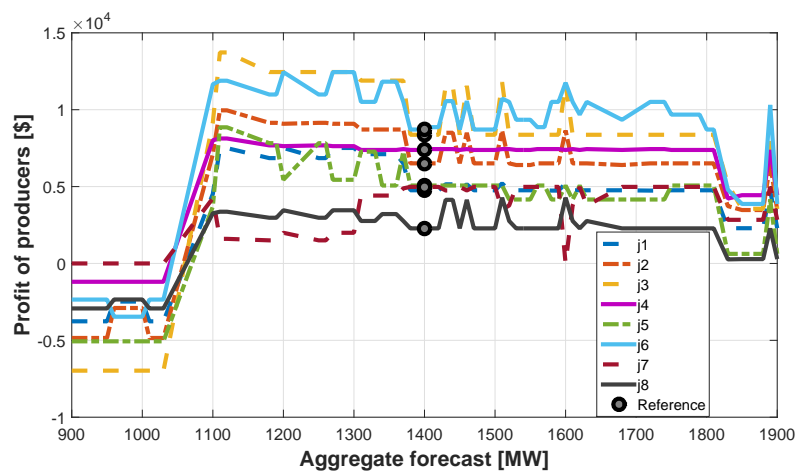

Fig. 8. Producers' profits for different aggregate forecast values considering uncertainty in wind power generation and real-time prices

aggregate forecasts of greater values than the reference, i.e., social welfare decreases. As anticipated, energy prices affect producers' profits as well. For small values of aggregate forecast, producers' profits are very low and in some cases even negative. Producers offer their power in lower prices, than the corresponding generation cost, mislead by erroneous aggregate forecasts causing them negative instead of positive profits.

Under such a setup, it is evident that the level of public aggregate forecast can indeed misguide producers' strategic behaviour. In turn, this has a major impact on social welfare, which is considerably decreased or increased, for overor under-forecasts. In this work, wind power was offered deterministically to the market based on a forecast. It is of our future interest to investigate how aggregate forecasts would impact the results under a stochastic market setup, as in [30] for example. Lastly, the outcomes of this study can contribute towards the discussion on the importance of sharing and publishing wind power forecasts on the benefit of market operation, initially discussed in [31]. Favored by ambitious plans for high quality market-related data, it is strongly believed that understanding the role of forecast information and their status, being public or private, can have a crucial impact on electricity market functioning. To this end, it is highly relevant to account for incentive-compatible mechanisms, such as mechanism design [32] or consensusbased distributed market clearing [33], which can elicit truthful decisions from market's parties, increasing the transparency of

the electricity market.

\section{APPENDIX}

\section{A. MPEC Formulation}

Lower-level problem (1d)-(1o) is continuous, linear and, therefore, convex. This allows bilevel problem (1) to be recast as a single-level MPEC through replacing the lowerlevel problem by its Karush-Kuhn-Tucker (KKT) optimality conditions, as presented below:

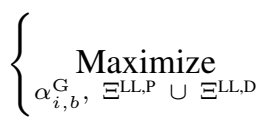

subject to:

(1b), (1c) and (1e)

$\alpha_{i, b}^{\mathrm{G}}-\lambda^{\mathrm{DA}}+\bar{\phi}_{i}^{\mathrm{G}}-\underline{\phi}_{i}^{\mathrm{G}}+\bar{\tau}_{i, b}-\underline{\tau}_{i, b}=0 \forall i, \forall b$

$-\lambda_{d}^{\mathrm{D}}+\lambda^{\mathrm{DA}}+\bar{\psi}_{d}-\underline{\psi}_{d}=0 \forall d$

$-\lambda^{\mathrm{DA}}+\bar{\sigma}_{l}-\underline{\sigma}_{l}=0 \forall l \in \mathcal{W}_{\mathcal{J}}$

$-\lambda^{\mathrm{DA}}+\bar{\sigma}^{\mathrm{MO}}-\underline{\sigma}^{\mathrm{MO}}=0$

$-\mu^{\mathrm{U}}+\bar{\rho}_{i}^{\mathrm{U}}-\underline{\rho}_{i}^{\mathrm{U}}+\bar{\phi}_{i}^{\mathrm{G}}=0 \forall i$

$-\mu^{\mathrm{D}}+\bar{\rho}_{i}^{\mathrm{D}}-\underline{\rho}_{i}^{\mathrm{D}}+\underline{\phi}_{i}^{\mathrm{G}}=0 \forall i$

$0 \leq\left(\sum_{i \in \mathcal{G}} r_{i}^{\mathrm{U}}-\gamma \sum_{d \in \mathcal{D}} \bar{P}_{d}^{\mathrm{D}}-\delta \sum_{l \in \mathcal{W}} \bar{W}_{l}\right) \perp \mu^{\mathrm{U}} \geq 0$

$0 \leq\left(\sum_{i \in \mathcal{G}} r_{i}^{\mathrm{D}}-\gamma \sum_{d \in \mathcal{D}} \bar{P}_{d}^{\mathrm{D}}-\delta \sum_{l \in \mathcal{W}} \bar{W}_{l}\right) \perp \mu^{\mathrm{D}} \geq 0$

$0 \leq r_{i}^{\mathrm{U}} \perp \underline{\rho}_{i}^{\mathrm{U}} \geq 0 \forall i$

$0 \leq\left(\bar{R}_{i}^{\mathrm{U}}-r_{i}^{\mathrm{U}}\right) \perp \bar{\rho}_{i}^{\mathrm{U}} \geq 0 \forall i$

$0 \leq r_{i}^{\mathrm{D}} \perp \underline{\rho}_{i}^{\mathrm{D}} \geq 0 \forall i$

$0 \leq\left(\bar{R}_{i}^{\mathrm{D}}-r_{i}^{\mathrm{D}}\right) \perp \bar{\rho}_{i}^{\mathrm{D}} \geq 0 \forall i$

$0 \leq p_{i, b}^{\mathrm{G}} \perp \underline{\tau}_{i, b} \geq 0 \forall i, \forall b$

$0 \leq\left(\bar{P}_{i, b}^{\mathrm{G}}-p_{i, b}^{\mathrm{G}}\right) \perp \bar{\tau}_{i, b}^{\mathrm{G}} \geq 0 \forall i, \forall b$

$0 \leq\left(\sum_{b \in B_{i}} p_{i, b}^{\mathrm{G}}-r_{i}^{\mathrm{D}}\right) \perp \underline{\phi}_{i}^{\mathrm{G}} \geq 0 \forall i$

$0 \leq\left(\sum_{b \in B_{i}} \bar{P}_{i, b}^{\mathrm{G}}-\sum_{b \in B_{i}} p_{i, b}^{\mathrm{G}}-r_{i}^{\mathrm{U}}\right) \perp \bar{\phi}_{i}^{\mathrm{G}} \geq 0 \forall i$

$0 \leq p_{d}^{\mathrm{D}} \perp \underline{\psi}_{d} \geq 0 \forall d$

$0 \leq\left(\bar{P}_{d}^{\mathrm{D}}-p_{d}^{\mathrm{D}}\right) \perp \bar{\psi}_{d} \geq 0 \forall d$

$0 \leq p_{l}^{\mathrm{W}} \perp \underline{\sigma}_{l} \geq 0 \forall l \in W_{\mathcal{J}}$

$0 \leq\left(F_{l}-p_{l}^{\mathrm{W}}\right) \perp \bar{\sigma}_{l} \geq 0 \forall l \in W_{\mathcal{J}}$

$0 \leq \sum_{l \notin \mathcal{W}_{\mathcal{J}}} p_{l}^{\mathrm{W}} \perp \underline{\sigma}^{\mathrm{MO}} \geq 0$

$0 \leq\left(F^{\mathrm{MO}}-\sum_{l \notin \mathcal{W}_{\mathcal{J}}} p_{l}^{\mathrm{W}}-\sum_{l \in \mathcal{W}_{\mathcal{J}}} F_{l}\right) \perp \bar{\sigma}^{\mathrm{MO}} \geq 0$ 
MPECs (3), one per producer, are non-linear due to the following two sources of non-linearities:

- The bi-linear terms $\lambda^{\mathrm{DA}} p_{i, b}^{\mathrm{G}}, \lambda^{\mathrm{DA}} p_{l}^{\mathrm{W}}, \mu^{\mathrm{U}} r_{i}^{\mathrm{U}}$ and $\mu^{\mathrm{D}} r_{i}^{\mathrm{D}}$ included in the objective function (3a).

- Complementarity conditions (3i)-(3x).

The bi-linear terms inside the objective function are linearized based on an approach without approximation, deploying the strong duality theorem and mathematical expressions (3c)-(3x), as in [24]. Finally, complementarity conditions are linearized based on an SOS1-based approach [34], [35] but at the cost of introducing a set of auxiliary SOS1 variables.

\section{B. Problem Formulation Considering Real-Time Stage Sce-} narios

Considering scenarios for the actual wind power generation and for the RT prices, the formulation of model (1) is transformed into (4) below:

$$
\begin{aligned}
& \left\{\begin{array}{c}
\text { Maximize } \\
\alpha_{i, b}^{\mathrm{G}}, p_{l, \omega}^{\mathrm{W}, \mathrm{RT}}, \Xi^{\mathrm{LL}, \mathrm{P}} \cup \Xi^{\mathrm{LL}, \mathrm{D}}
\end{array}\right. \\
& \sum_{i \in \mathcal{G}_{\mathcal{J}}} \sum_{b \in B_{i}}\left(\lambda^{\mathrm{DA}}-C_{i, b}^{\mathrm{G}}\right) p_{i, b}^{\mathrm{G}} \\
& \quad+\sum_{l \in \mathcal{W}_{\mathcal{J}}}\left(\lambda^{\mathrm{DA}} p_{l}^{\mathrm{W}}\right)+\sum_{i \in \mathcal{G}_{\mathcal{J}}}\left(\mu^{\mathrm{U}} r_{i}^{\mathrm{U}}+\mu^{\mathrm{D}} r_{i}^{\mathrm{D}}\right) \\
& \quad-\sum_{\omega \in \Omega} \pi_{\omega} \sum_{s \in \mathcal{S}} \pi_{s}^{\lambda}\left[\sum_{l \in \mathcal{W}_{\mathcal{J}}} \lambda_{s}^{\mathrm{RT}} p_{l, \omega}^{\mathrm{W}, \mathrm{RT}}\right]
\end{aligned}
$$

subject to:

$$
\text { (1b) }-(1 \mathrm{c})
$$$$
P_{l, \omega}^{\mathrm{W}, \mathrm{P}}+p_{l, \omega}^{\mathrm{W}, \mathrm{RT}}=p_{l}^{\mathrm{W}} \quad \forall l \in \mathcal{W}_{\mathcal{J}}, \forall \omega
$$

where $\lambda^{\mathrm{DA}}, p_{l}^{\mathrm{W}}$ and $p_{i, b}^{\mathrm{G}} \forall i \in \mathcal{G}_{\mathcal{J}}$,

$$
\forall l \in \mathcal{W}_{\mathcal{J}}, \forall b \in \arg \{
$$$$
\underset{\Xi^{\mathrm{LL}, \mathrm{P}}}{\operatorname{maximize}} \sum_{d \in \mathcal{D}} \lambda_{d}^{\mathrm{D}} p_{d}^{\mathrm{D}}-\sum_{i \in \mathcal{G}} \sum_{b \in B_{i}} \alpha_{i, b}^{\mathrm{G}} p_{i, b}^{\mathrm{G}}
$$

subject to:

$$
\begin{aligned}
& \text { (1e) }-(10)\} \\
& \} \forall \mathcal{J}
\end{aligned}
$$

where constraint (4c) ensures that the power imbalance due to the difference between the DA scheduled wind power and the actual generated one under scenario $\omega$, is adjusted in RT by the traded RT wind power. A positive value of $p_{l, \omega}^{\mathrm{W}, \mathrm{RT}}$ indicates that wind power production is lower than the scheduled one in DA market, incurring an additional cost for the producer.

\section{Computational Issues}

For the simulations of this paper we have used CPLEX under GAMS on a Windows 8.1, 64-bit operating system with 2 cores processor running at $2.4 \mathrm{GHz}$ and $12 \mathrm{~GB}$ of RAM. The total computational time for the whole case-study was 720 s. Furthermore, for the cases where equilibrium was found ( 82 out of 101) it took less than 5 iterations to find the equilibrium. The computational time depends highly on whether an equilibrium is found or if the process terminates after maximum number of iterations, the latter requiring more time. The convergence tolerance was set at $\epsilon=0.3$ and the maximum number of iterations $c_{\max }=10$. In this paper, we use an iterative diagonalization approach which is simple but at the risk of not converging in the predefined number of iterations. One sort of alternatives is to augment the current diagonalization technique by increasing the number of iterations and/or providing different starting points. Another alternative is to use non-iterative equilibrium solution techniques (e.g., in [26]), but at the cost of increased complexity. Lastly, the setup that was chosen for this study allows us to get an insight into the role of public aggregate forecasts in electricity markets without constructing a complicated tool. Scaling the problem up to a $n$-player setup would lead to an agent-based analysis with similar qualitative results but increased computational complexity.

\section{REFERENCES}

[1] "Electricity Market Regulators, EU countries and stakeholders discuss EU Internal Electricity Market," https://ec.europa.eu/energy/en/news/ electricity-market-regulators-member-states-and-stakeholders-discusseuropean-internal/, 2015, [Online; accessed 28-Oct-2016].

[2] "XXVIII European Electricity Regulatory Forum," https://ec.europa.eu/ energy/sites/ener/files/documents/Conclusions_Final_FF_June2015.pdf, 2015, [Online; accessed 28-Oct-2016].

[3] "Commission Regulation (EU) no 543/2013," http://eurlex.europa.eu/legal-content/EN/TXT/PDF/?uri=CELEX:32013R0543\& qid $=1477468359304 \&$ from $=E N$, 2013, [Online; accessed 28-Oct-2016].

[4] "Elia," http://www.elia.be/en/grid-data/power-generation/wind-power, [Online; accessed 28-Oct-2016].

[5] "Transparency of Data about the Electricity Market," http://www.elia. be/en/grid-data/transparency/, 2013, [Online;accessed 28-Oct-2016].

[6] "ENTSO-E transparency platform," https://transparency.entsoe.eu/, [Online; accessed 28-Oct-2016].

[7] "Independent System Operator of New England," http://www.isone.com/isoexpress/web/reports/operations/-/tree/seven-day-windpower-forecast, [Online; accessed 28-Oct-2016].

[8] "Midcontinent independent system operator," https://www. misoenergy.org/MarketsOperations/RealTimeMarketData/Pages/ DayAheadWindForecast.aspx, [Online; accessed 28-Oct-2016].

[9] "Alberta electric system operator," http://www.aeso.ca/gridoperations/ 18286.html, [Online; accessed 28-Oct-2016].

[10] J. B. Cardell, C. C. Hitt, and W. W. Hogan, "Market power and strategic interaction in electricity networks," Resource and Energy Economics, vol. 19, no. 12, pp. 109-137, 1997.

[11] B. F. Hobbs, C. B. Metzler, and J. S. Pang, "Strategic gaming analysis for electric power systems: an MPEC approach," IEEE Trans. Power Syst., vol. 15, no. 2, pp. 638-645, May 2000.

[12] H. Pandzic, A. J. Conejo, and I. Kuzle, "An EPEC approach to the yearly maintenance scheduling of generating units," IEEE Trans. Power Syst. vol. 28, no. 2, pp. 922-930, May 2013.

[13] S. Steffensen and M. Bittner, "Relaxation approach for equilibrium problems with equilibrium constraints," Computers \& Operations Research, vol. 41, pp. 333-345, 2014.

[14] "Technology roadmap: Energy storage," International Energy Agency, 2014.

[15] G. Giebel, R. Brownsword, G. Kariniotakis, M. Denhard, and C. Draxl, "The state-of-the-art in short-term prediction of wind power: A literature overview," Tech. Rep., 2011.

[16] C. J. Dent, J. W. Bialek, and B. F. Hobbs, "Opportunity cost bidding by wind generators in forward markets: Analytical results," IEEE Trans. Power Syst., vol. 26, no. 3, pp. 1600-1608, Aug. 2011.

[17] S. N. Singh and I. Erlich, "Strategies for wind power trading in competitive electricity markets," IEEE Transactions on Energy Conversion, vol. 23, no. 1, pp. 249-256, Mar. 2008.

[18] G. N. Bathurst, J. Weatherill, and G. Strbac, "Trading wind generation in short-term energy markets," IEEE Power Engineering Review, vol. 22, no. 7, pp. 54-54, Jul. 2002. 
[19] A. Fabbri, T. G. S. Roman, J. R. Abbad, and V. H. M. Quezada, "Assessment of the cost associated with wind generation prediction errors in a liberalized electricity market," IEEE Trans. Power Syst., vol. 20, no. 3, pp. 1440-1446, Aug. 2005.

[20] M. Zugno, J. M. Morales, P. Pinson, and H. Madsen, "Pool strategy of a price-maker wind power producer," IEEE Trans. Power Syst., vol. 28, no. 3, pp. 3440-3450, Aug. 2013.

[21] L. Baringo and A. J. Conejo, "Strategic offering for a wind power producer," IEEE Trans. Power Syst., vol. 28, no. 4, pp. 4645-4654, Nov. 2013.

[22] A. A. S. de la Nieta, J. Contreras, J. I. Munoz, and M. O'Malley, "Modeling the impact of a wind power producer as a price-maker," IEEE Trans. Power Syst., vol. 29, no. 6, pp. 2723-2732, 2014.

[23] T. Dai and W. Qiao, "Optimal bidding strategy of a strategic wind power producer in the short-term market," IEEE Transactions on Sustainable Energy, vol. 6, no. 3, pp. 707-719, 2015.

[24] C. Ruiz, A. J. Conejo, and Y. Smeers, "Equilibria in an oligopolistic electricity pool with stepwise offer curves," IEEE Trans. Power Syst., vol. 27, no. 2, pp. 752-761, May 2012.

[25] C. Ruiz, S. J. Kazempour, and A. J. Conejo, "Equilibria in futures and spot electricity markets," Electric Power Systems Research, vol. 84, no. 1, pp. 1-9, 2012.

[26] S. J. Kazempour and H. Zareipour, "Equilibria in an oligopolistic market with wind power production," IEEE Trans. Power Syst., vol. 29, no. 2, pp. 686-697, Mar. 2014.

[27] T. Dai and W. Qiao, "Finding equilibria in the pool-based electricity market with strategic wind power producers and network constraints," IEEE Trans. Power Syst., to be published.

[28] M. R. Hesamzadeh and M. Yazdani, "Transmission capacity expansion in imperfectly competitive power markets," IEEE Trans. Power Syst., vol. 29, no. 1, pp. 62-71, 2014.

[29] Reliability Test System Task Force, "The IEEE reliability test system1996," IEEE Trans. Power Syst., vol. 14, no. 3, pp. 1010-1020, Nov./Dec. 1999.

[30] J. M. Morales, A. J. Conejo, K. Liu, and J. Zhong, "Pricing electricity in pools with wind producers," IEEE Trans. Power Syst., vol. 27, no. 3, pp. 1366-1376, Aug. 2012.

[31] L. Exizidis, S. J. Kazempour, P. Pinson, Z. de Greve, and F. Vallee, "Sharing wind power forecasts in electricity markets: A numerical analysis," Appl. Energy, vol. 176, pp. 65-73, 2016.

[32] T. W. Haring, D. S. Kirschen, and G. Andersson, "Incentive compatible imbalance settlement," IEEE Trans. Power Syst., vol. 30, no. 6, pp. 3338 3346, Nov 2015.

[33] Y. Zhang and G. B. Giannakis, "Distributed stochastic market clearing with high-penetration wind power," IEEE Trans. Power Syst., vol. 31, no. 2, pp. 895-906, Mar. 2016.

[34] S. Siddiqui and S. A. Gabriel, "An SOS1-based approach for solving MPECs with a natural gas market application," Networks and Spatial Economics, vol. 13, no. 2, pp. 205-227, 2013.

[35] S. A. Gabriel, A. J. Conejo, J. D. Fuller, B. F. Hobbs, and C. Ruiz, Complementarity Modeling in Energy Markets, New York, NY, USA: Springer, 2012.

Lazaros Exizidis (StM'14) received his diploma in Electrical and Computer Engineering from the Aristotle University of Thessaloniki. Since 2013 he is a PhD Candidate in Electrical Engineering at the University of Mons. His research interests include wind power forecasting, optimization in power systems and electricity markets.

Jalal Kazempour (M'14) is an assistant professor at the Department of Electrical Engineering, Technical University of Denmark, Kgs. Lyngby, Denmark. He received his Ph.D. degree in electrical engineering from University of Castilla-La Mancha, Ciudad Real, Spain, in 2013. His research interests include power systems, electricity markets, optimization, and its applications to energy systems.

Pierre Pinson (M'11-SM'13) received the M.Sc. degree in applied mathematics from the National Institute for Applied
Sciences (INSA Toulouse, France) and the Ph.D. degree in energetics from Ecole des Mines de Paris (France). $\mathrm{He}$ is a Professor at the Technical University of Denmark (DTU), Centre for Electric Power and Energy, Department of Electrical Engineering, also heading a group focusing on Energy Analytics \& Markets. His research interests include among others forecasting, uncertainty estimation, optimization under uncertainty, decision sciences, and renewable energies. Prof. Pinson acts as an Editor for the International Journal of Forecasting, and for Wind Energy.

Zacharie De Gréve (M'12) holds an Electrical and Electronics Engineering degree from the Faculty of Engineering of Mons, Belgium (2007). He has been a research fellow of the Belgian Fund for Research (F.R.S/FNRS) until 2012, when he got the PhD degree in Electrical Engineering, from the University of Mons. He is now a research and teaching assistant at the Electrical Power Department of the same university. His research interests include the numerical modeling of electromagnetic fields, as well as the integration of renewable energies in power electrical networks.

François Vallée (M'09) received his degree in civil electrical engineering and the Ph.D. degree in electrical engineering from Faculté Polytechnique de Mons (Belgium) in 2003 and 2009, respectively. He is currently an Associate Professor in the Power Electrical Engineering Unit of Faculté Polytechnique, University of Mons (Belgium). His fields of interest mainly include wind generation modelling and electrical network reliability in presence of dispersed generation. 